Forthcoming: Chapter 32, Handbook of Communication Neuroscience, Routledge Press.

\author{
Virtual Reality for Communication Neuroscience \\ Bradly Alicea ${ }^{1,2}$ \\ ${ }^{1}$ Orthogonal Research and Education Lab, bradly.alicea@outlook.com \\ ${ }^{2}$ OpenWorm Foundation
}

\begin{abstract}
Virtual Reality (VR) and human interaction with virtual worlds can greatly enhance communication neuroscience research. VR provides opportunities for novel approaches to empirical investigation. This chapter will discuss why VR and the varieties of virtual experience is of interest to communication neuroscientists. This leads to a discussion of multiple issues related to experimental implementation and cognition, and ways VR is useful to communication neuroscience. This includes multiple cognitive effects that provide opportunities for advancing research.
\end{abstract}

Keywords: Cognitive Neuroscience, Virtual Reality, Communication Theory

\title{
Introduction
}

Virtual Reality (VR) is an emerging and useful tool for behavioral scientists, in general, and communication neuroscience, more specifically. The point of VR is to create simulations that correspond either to reality as it has been experienced or to the world of sensory experience as it might be. Given the right mix of technology and representation, we can simulate the feeling of being in a race car or riding a rollercoaster. We can transport the viewer to the surface of distant planets or other alternative realities with ease. Most important, we can control the stimulus and guide the experience to something that is quantifiable using contemporary experimental methods.

This chapter will discuss why VR is interesting to communication neuroscientists. One of these reasons involves the varieties of virtual experience available to the experimental participant. We will also engage in a discussion of the pros and cons related to using VR in the study of cognitive processes. There are also a number of ways $V R$ is useful to communication neuroscience in terms of both stimulus design and psychological effects. Finally, we will discuss naturalistic investigations, the development of open cognitive models, and potential future research directions. 


\section{Basic Questions}

This section will discuss a host of questions and issues related to introducing readers to the world of using VR to study communication neuroscience. The basic questions include why VR is interesting to neuroscientists and how VR works in terms of technology and media content. Two main issues are also discussed: the technical components of a VR system that is used for research, and the varieties of virtual experience that result from exposure to a virtual environment.

\section{Why Is VR Interesting?}

VR has been part of the behavioral research landscape for decades, although its widespread adoption has been much more recent. We can use VR to study a wide range of cognitive functions, including spatial cognition (Hardiess et al., 2015), skill learning and expertise (Thompson et al., 2013), social connectivity (Tarr et al., 2018), cognitive performance and training (Bohil et al., 2011), and attention (Weber et al., 2018). It is easy to see the advantages of using VR in an experimental context, as VR also provides a high-resolution, multimodal stimulus with good ecological validity (Bohil et al., 2011; Reggente et al., 2018). This enables new types of experimental design, such as the freeviewing condition (Bartels \& Zeki, 2004) and other naturalistic approaches to the study of cognition and media (Hasson \& Honey, 2012).

Yet there is a more fundamental advantage of using VR systems. According to the media equation hypothesis (Reeves \& Nass, 1996), high-resolution and interactive media are a surrogate of the natural world. For example, one can use a virtual avatar to explore a virtual model of a city park. Whereas the avatar's movements are indirectly initiated using a controller, the human can nevertheless be mentally invested in the behavior of the avatar. In addition, there is an equivalence in terms of complex skill learning between the real and simulated environments. Such an outcome has been observed for a rowing simulation that includes physical cues (Rauter et al., 2013). Moreover, people react to a familiar-looking virtual simulation without conscious effort (Reeves \& Nass, 1996), much as they would respond to a well-known natural scene (Martens et al., 2012). This type of immersive telepresence (Slater \& Sanchez-Vives, 2016) allows us to study both seamless cognitive experiences and controlled dissection of cognitive processes.

\section{How Does VR Work?}

VR takes advantage of our ability to observe action, plan for the future, and synthesize sensorimotor inputs. Although virtual worlds are often immersive, they are also highly interactive. According to McLuhan (1964), VR can generally be categorized as 
both "hot" and "cool" media. Hot media engage the senses briefly and intensely, often with one sensory input (vision) dominating all of the others. Yet VR also has components of "cool media," notably in the case of narrative-based video games that provide opportunity for extended engagement. Truly immersive VR experiences can stimulate multiple senses simultaneously, allowing for a greater degree of virtual-to-real world equivalence. In terms the medium's inherent semantic content (McLuhan, 1964), VR is similar to movies in that it serves as a transition from static images and linear action to configurations of images and the potential for non-linear action. The specifics of how this occurs depends upon the virtual stimulus itself and how it is presented to the nervous system.

\section{Technical Components}

The technical components of VR are also flexible, and involve interfaces specific to each sense that is engaged. A wide range of visual and non-visual stimuli can be delivered to the nervous system, from vibratory sensations along the arms and legs to simulated hands in the field of vision. These stimuli can in turn be delivered using a number of perspectives, from first-person shooter video games to race car simulations with motion cues.

Visual information can be displayed in a Cave-Automatic Virtual Environment (CAVE) screen, a Head Up Display (HUD), or a Head Mounted Display (HMD). Auditory information can be delivered using a pair of earphones. Haptic information (touch) can be delivered using pinch gloves or a vibrating video game controller.

Application of technology. Technical choices will also depend on what the experimenter wants to simulate and the physical constraints of the experimental setting. In an $\mathrm{fMRI}$ scanner, specialized equipment will be necessary. Due to the requirements of motion capture and potential for artifact generation, normal operation of the VR interface may constrain mobility (Figure 1). In general, coupling VR and neural measurement provides a number of challenges. Neural measurement techniques such as fNIR or EEG do not require highly specialized VR configurations. However, they can be affected by motion artifact, requiring users to limit their limb and trunk movements.

VR systems can also be affected by motion artifact, particularly during the registration of body movements with positions of objects in the virtual environment. Fortunately, the limitations of neuroimaging technique and VR system configuration can be dealt with in tandem. Related to this, a major challenge of integrating neuroimaging and VR technologies is the synchronization of action in the simulation with continuous 
measurements of neural activity. Although such synchronization is difficult, it does provide a rich and highly informative dataset.

\section{Varieties of Experience}

Virtuality encompasses more than simply a collection of interfaces and content. According to Steuer (1992), VR is defined as any environment in which an individual experiences telepresence. Thus, experience is driven not by the act of simulation itself, but by some undefined cognitive function. The experiential response is elicited by stimulus form: virtual stimuli must be not only realistic, but realistic in context. For our purposes, VR encompasses three major types of simulation: immersive VR, augmented reality $(A R)$, and mixed reality $(M R) . V R, A R$, and $M R$ are three variations on modifying a world of physical objects in a physical world. VR can be described as virtual objects in a virtual world. By contrast, AR involves virtual objects in a physical world. As the inverse of $A R, M R$ presents viewers with physical objects in a virtual world (see Table 1).

Simulation and experience taxonomy. I propose that the broad variety of experiences generated by a VR simulation can be distilled to two categories: augmentative and narrative (Table 1). Augmentative experience is the presentation of objects in locational context or depth perspective. A roller coaster simulation is augmentative in that it provides a human in a non-moving room with the feeling of being on a roller coaster. In contrast, narrative experience involves engagement with a story or a series of events linked by meaning. This includes both action sequences in video games and movies and stories that are based on a sequence of events. Augmentative and narrative experience can also merge to produce a coherent experience. In $\mathrm{VR}$, we can combine an interactive narrative with augmentative features such as simulated motion or an increased flow of time.

Table 1. Major types of simulation and two types of experience in VR.

\begin{tabular}{|c|c|c|c|}
\hline & Virtual Reality (VR) & $\begin{array}{c}\text { Augmented Reality } \\
\text { (AR) }\end{array}$ & Mixed Reality (MR) \\
\hline $\begin{array}{c}\text { Type of } \\
\text { Virtuality }\end{array}$ & $\begin{array}{c}\text { Virtual Objects, } \\
\text { Virtual World }\end{array}$ & $\begin{array}{c}\text { Virtual Objects, } \\
\text { Physical World }\end{array}$ & $\begin{array}{c}\text { Physical Objects, } \\
\text { Virtual World }\end{array}$ \\
\hline Augmentative & Pilot training & Annotated City Map & $\begin{array}{c}\text { Outer Space } \\
\text { Simulation }\end{array}$ \\
\hline Narrative & Fantasy games & $\begin{array}{c}\text { Adventure Games } \\
\text { (Pokemon) }\end{array}$ & List of Instructions \\
\hline
\end{tabular}


The combination of augmentative and narrative experience combine into something often referred to as telepresence. Telepresence involves both the feeling of being transported to the actual environment by the virtual world (Gerrig, 1993) and how a certain experience feels in general (Rheingold, 1991). The feeling of realness comes from the features of the medium, or media form (Kim \& Biocca, 1997). Telepresence can also involve competition between real-world and virtual stimuli to create a perceptual illusion (Biocca \& Delaney, 1995). Variables such as visual angle of the display or illumination level of the room can lead to effects such as sensory saturation and sensory suppression, respectively (Kim \& Biocca, 1997). These can enhance the level of augmentative experience directly and the level of narrative experience indirectly. This sets the stage for numerous advantages and disadvantages to the use of VR technology as a real-world analogue.

\section{Advantages and Limitations of the VR Medium}

In this section, the advantages and limitations of using VR in communication neuroscience will be discussed. The advantages include ease of use and experimental implementation, the ability to take advantage of perceptual affordances, and the ability to trigger a neuroplastic response with neuroimaging-friendly stimuli. Limiting factors include the presence of perceptual gaps and the need to achieve coherent perceptual states for maximum experimental effect.

\section{Advantages of the VR medium}

There are several advantages to using VR in an experimental setting. These advantages include ease of use, controllability, and customization. Unlike a sequential movie or static set of experimental images, the stimulus can be explored in a combinatorial manner (McLuhan, 1964). This allows not only for participants to interact with the stimulus more naturally, but also for the stimulus to be reconfigured in an experiment-specific manner. Hasson et al. (2008) discuss the use of neuroimaging to study how people respond to films, in particular the continuous, sequential nature of the medium. Using a specialized type of analysis (inter-subject correlation), it becomes easy to examine the effectiveness of a media stimulus on a common cognitive process without the bias of subjective experience (Hasson et al., 2008).

Ease of use. VR environments are easily constructed and become highly customizable at relatively low cost. Widely available software tools and programming languages provide an experimenter with many possible designs for environments, such as a maze or a racetrack. Not only are these environments highly reconfigurable, they also exhibit a 
high degree of replicability across participants, conditions, and experiments. As objectbased simulations of real-world phenomena, VR worlds can be controlled simply by changing the programming. This enables a high degree of experimental control. The programmer can determine how the environment is represented across conditions and trails, in addition to reducing experimental noise by guiding the participant toward specific pathways and outcomes.

Experimental implementation. VR systems can also be easy to implement in an experimental setting. For example, the ability to reconfigure virtual scenes in an experimentally reproducible manner (for an example for simple visual tasks, see Saleh et al., 2013) ultimately results in increase ecological validity, thus making the application of naturalistic methods more reliable as well. There are also advantages that enable unique models of experimental investigation. This allows us to ask compelling questions not typically possible using more traditional methods. For example, a free-viewing condition can be implemented without the potential confounds of itinerant behavior and uncharacterized environmental interactions. We can also explore phenomena such as proprioceptive and vestibular inputs during spatial cognition (for an example using path integration, see Chance et al., 1998).

Affordances. Another advantage to increased experimental control involves the ability to use the principle of perceptual affordances (Good, 2007). Affordances are features of the object and/or environment that have significance or even meaning to humans. The handle of a door or coffee mug is one such example: handles are interpreted as a place to manipulate an object. The ubiquity of perceptual affordances in VR environments allows an experimenter to guide perception and action in a desired way, or even uncover behaviors not easily observed in the real-world. We will discuss the critical role affordances play in determining perception and action as a unique mode of interaction. Given the flexible control of virtual environments, affordances can be enhanced, deemphasized, or associated with unexpected places to elicit behaviors such as confusion or exploration.

Neuroplasticity and longer-term changes. Yet a fourth advantage of using VR as an experimental stimulus is the ability to find people with different levels of skill in terms of their experience with the environment. Furthermore, participants can be trained on a specific VR system (e.g., a video game) to acquire expertise. Although controversial (see Simons et al., 2016), the acquisition of expertise provides the brain with enhanced or protective function that improves cognitive performance. A number of studies have been done that assess differences between video game players (those with expertise) 
versus non-video game players (those without expertise). Such effects are heavily dependent on the type of video game, the degree of expertise held by the experimental group, and the experimental design, and can be subject to interpretation (Hambrick et al., 2014). Nevertheless, it has been demonstrated that performance improvements in visual attention and spatial learning (Green \& Bavelier, 2007, 2012) as well as related anatomical differences (West et al., 2017) are possible long-term effects of repeated engagement with VR environments.

Neuroimaging-friendly stimuli. For brain scientists, all of these positives are underscored by the potential for neuroimaging-friendly implementation. Given specialized interfaces, VR can be easily interacted with in an fMRI scanner or while wearing an EEG cap. This allows us to study a number of cognitive functions and effects in the brain that would not be possible without VR. VR can also be combined with neuromodulation techniques to produce closed-loop adaptive feedback. In the fields of neurorehabilitation (Teo et al., 2016) and human-computer interface design (Huster et al., 2014), such systems have been used to improve cognitive and physical performance. Using other combinations of measurement, virtuality, and direct neural input, we might also be able to understand VR-specific behaviors and outcomes that have not been previously been characterized.

\section{Limitations of the VR medium}

There are a number of challenges and limitations to VR technologies that constrain their potential, at least until further research and technological development advance to a suitable point. The first such limitation is that performance in a virtual environment is not always equivalent to real-world performance. One rather contentious issue in the Human Factors literature is the equivalence of virtual and real-world training for tasks such as piloting an airplane (Myers et al., 2018). Virtual stimuli can also be exaggerated either in the timing of their delivery or providing ambiguity in terms of their actual size. Although we often take the juxtaposition of perceptual inputs for granted in real-world contexts, their specificity is a key design feature of virtual worlds. Successful immersion in a virtual environment involves three properties: inclusive, extensive, and incongruence (Bystrom et al., 1999). These properties can enhance (or limit) perceptual fluidity, which in turn is affected by the absence of perceptual gaps and emergence of coherent percepts.

Perceptual gaps. Perceptual gaps are both uncontrollable and poorly understood. Whereas unintended perceptual gaps can result from latency in the delivery of the stimulus (e.g., video frame rate lag), they can also arise from cognitive processing itself. 
One example of a perceptual gap has to do with the phenomenon of presence. Presence involves a number of cognitive functions and deeply influences how we judge the "realness" of a given stimulus (see the plausibility illusion of Slater, 2009). Although not the same as immersion, presence involves subconscious reality judgements with respect to the stimulus. Rather, presence arises as a consequence of whether or not the current experience is credible enough to engage with (Baños et al., 2000).

Perceptual gaps can also be found in and characterized by formal psychological effects related to the use of VR. One such example is the uncanny valley effect (Cheetham, 2017). The uncanny valley involves affective ambiguity toward a human-like stimulus (Urgen et al., 2018). A much different example involves psychophysical effects such as the visually based prism adaptation (Chapman et al., 2010), which may result in significant misalignment between acquired neural representations of the action and the current state of the environment. One candidate cause of perceptual gaps is a lack of explicit 3-D feedback between the novelty of a virtual environment and the nervous system (Fulvio \& Rokers, 2017). As visual, auditory, and haptic perception are all part of a complex stimulus, perceptual gaps can also occur in a way that allows not only for interference but also for the construction of an alternate reality. This can be seen in the cutaneous rabbit illusion (Geldard, 1982), which involves rapid and successive stimulation of closely spaced points along the skin. This leads to the illusion of a rabbit crawling up the arm. Although the multimodal aspects of a realistic simulation are difficult to synchronize, understanding how they are experienced in different contexts is of primary importance.

Coherent percepts and novel behaviors. Another limitation (or potential design principle) involves the relationship between mental coherence and presentation of the virtual stimulus. Perception of objects in natural scenes often involves multisensory processing, even when the input to non-visual sensory systems is subtle. Coherent perception of events and awareness of experience relies on spatiotemporal synchronization between sensory modalities. This is known from both experimental decoupling of complex stimuli and multimodal fusion by specific neurons in the brain. When the different components of a complex stimulus are decoupled (e.g., the visual and auditory components are presented at different spatial locations), this has a direct effect on multimodal fusion. This in turn has a larger effect on the experience of sync (Huskey et al., 2018) and flow (Csikszentmihalyi, 1990), namely in the disruption of such continuous states.

\section{Fundamental Issues}


There are two fundamental issues for implementing VR in experimental contexts that will be highlighted here. Perception-action coupling and the definition of a virtuality brain network provide a set of initial assumptions ultimately translating into rigorous, reproducible studies.

\section{Perception-Action Coupling}

One fundamental issue for implementation of VR in experimental contexts is the role of perception-action coupling. Namely, can sensory stimulation be actionindependent? We can seek insight from the action selection literature to understand the linkages between sensory experience and decision-making (Barron et al., 2015), motor learning/control, and agency (Sidarus et al., 2015). We can also use computational models to understand these relationships better. Using an artificial life model, Seth (2007) defines action selection as a set of coupled sensorimotor processes. Overall, action selection allows us to prioritize our actions given a continuous flow of stimuli. Viewing the flow of perception from an ecological standpoint (Gibson, 2015) is essential to understanding the nature and dynamic aspects of virtual experience.

Another way to understand the link between cognition and virtual reality is through more formal cognitive modeling (Figure 1). Whereas cognitive modeling has been used to predict and explain multiple interacting components of human performance in a wide range of task environments, the role of higher order phenomena such as awareness and engagement has largely been avoided. One way to view a VRspecific cognitive model is to focus on the components of the hypothetical virtuality network.

\section{Toward a "Virtuality Network"}

It is unclear whether cognition in virtual environments is mediated by a combination of well-characterized cognitive functions or whether it requires a novel set of cognitive functions heretofore unknown. Much as there are attentional, emotional, and default activity networks in the brain, there might also be a "virtuality" network that mediates environmental features unique to VR (Figure 2).

The functional components of a virtuality network might involve interactions between premotor and motor cortex, spatial cognition centers, multimodal integration centers, and brain regions involved in object and face processing. Yet other types of functionality such as spatial navigation in high-dimensional artwork might also involve a mechanism related to abstract reasoning or consciousness. Alternatively, we might also 
learn something about the evolutionary cognitive substrate of virtuality by surveying the literature on the use of virtual reality in animal models (Alicea, 2015).

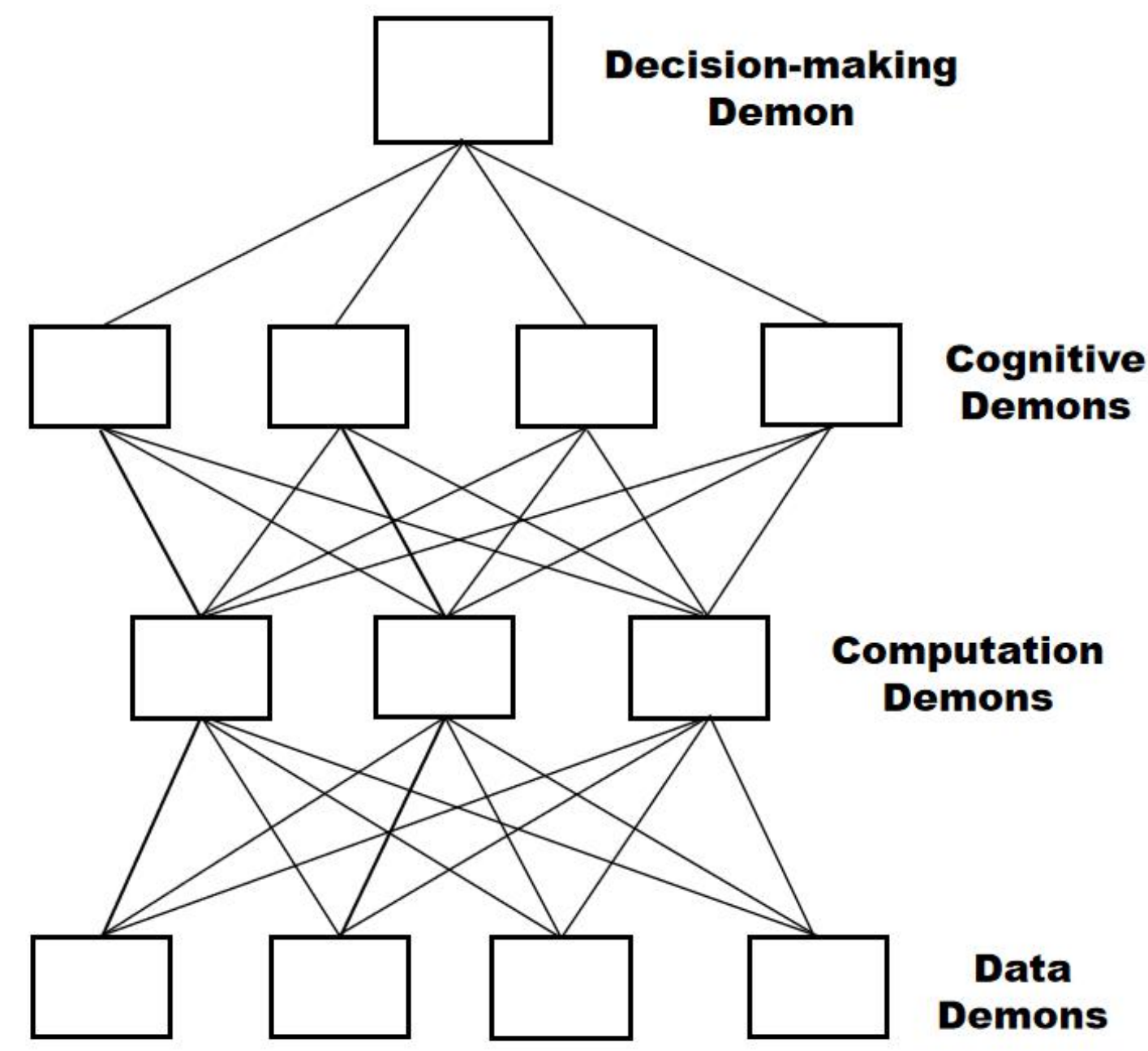

Figure 1. An example of a cognitive model based on the pandemonium model of Selfridge (1959). Layers include decision-making (top), cognitive demons (second from top), computation demons (second from bottom), and data demons (bottom).

\section{When Virtual Reality Becomes Useful}

To discuss how virtual reality becomes useful, several topical areas will be highlighted. These include but are not limited to: the necessary creation of new methods and phenomenology, the inclusion of sustained behavioral measurement and unique modes of interaction, and the enabling of unique behavioral modes. 


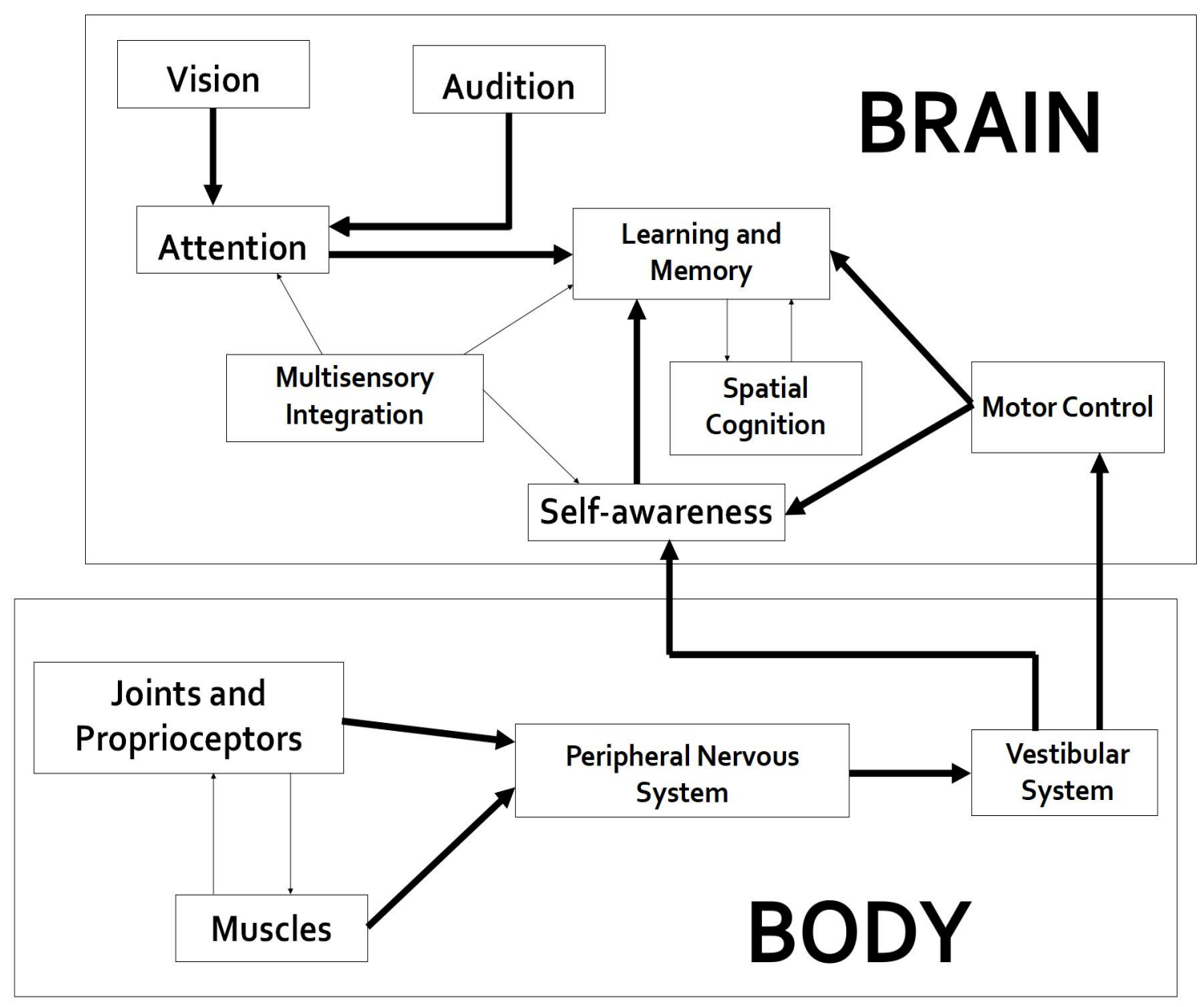

Figure 2. Model of a virtuality network as a putative cognitive model.

\section{New Methods and Phenomenology}

Aside from considering how VR can be advantageous (or deleterious) in measuring neurobehavior, it is quite useful for enabling both new methods and phenomenology. These include, but are not limited to, sustained behavioral measurement, unique modes of interaction, and unique modes of behavior. As we will see, a combination of ingenious methods and the unique features of virtual environments can provide an exciting new perspective on cognition and brain function.

Sustained behavioral measurement. From a quantitative standpoint, permitting participants to engage in behaviors such as navigation, aggression, and attentional focusing over long periods of time (more than a single trial or set of trials) has a number of advantages. Whereas specific behaviors can be segmented and classified for hypothesis testing, continuous media stimuli can reveal longer-term cumulative and 
emergent cognitive effects. Continuous measurement allows us to acquire time-series data, which requires tools such as frequency domain and dynamic causality analysis (Ozaki, 2012). Continuous data may also provide a window into a whole class of dynamical phenomena such as self-organized criticality, phase transitions, and metastability to be observed (Beggs, 2008; Kozma \& Freeman, 2017). More specifically, it allows for time-series data to be collected, which in turn allows for new types of analyses and contains information content not measurable as a set of discrete, randomized observations.

Unique modes of interaction. Aside from effects relevant to individual cognition, VR also enables people to interact in ways that are impossible in the physical world. When this is scaled up so that many people have access to the same content, it provides a unique window into social neuroscience by allowing us to understand how groups of people behave in a dynamic fashion. In particular, a method called hyperscanning (Balconi \& Vanutelli, 2017) can provide a link between human social interactions and the simultaneous imaging of multiple brains. One example comes from the practice of neuroeconomics, wherein hyperscanning is used to enable the dynamic monitoring of continuous and virtually-mediated economic transactions (Rangel et al., 2008). Overall, hyperscanning and other social measurement techniques hold much promise for communication neuroscience.

The use of affordances in VR object design also provides a means to introduce new semantic information into the medium. In particular, affordances provide signifiers within the VR environment (Norman, 2008) in a way that provides meaning and constructivist potential (Olson, 2017). Rather than providing opportunities for perceptual illusion, signifiers provide the symbolic glue for perception and action by clarifying the specific role of affordances in the environment. Affordances also provide physical cues to the individual from the virtual environment. According to Regia-Corte et al. (2013), affordances (in this case, a slanted surface) allow an individual to infer physical properties that support judgments that influence perception and action.

Unique modes of behavior. Ultimately, exposure to VR may lead to new behaviors. At a basic level, perceptual adaptations such as the prism effect (Chapman et al., 2010) are well-known aftereffects of exposure to VR environments. According to Gonzalez-Franco and Lanier (2017), there are three types of illusion associated with VR: place, embodiment, and plausibility. The place illusion occurs when the virtual environment transports the user to a new spatial location. It could be a familiar location or an exotic one. The embodiment illusion involves experiencing the virtual environment either in a 
disembodied form or as an avatar. As the intersection of both the place and embodiment illusion, the plausibility illusion involves self-examination as to whether or not an experience is actually happening. In this way, the plausibility illusion overlaps with some of the theoretical issues faced by the presence community.

There are other perceptual illusions that are enabled a consequence of the embodiment illusion. One of these is called body transfer, and is exemplified by the rubber (sometimes called marble) hand illusion (Senna et al., 2014). In this example, body transfer occurs when a participant begins to treat a virtual hand as part of his or her own body. While this illusion becomes behaviorally manifest by the artificial hand becoming equivalent to an additional appendage, the phenomenon is represented in the brain as a complex set of multimodal interactions (Ehrsson et.al, 2005). Returning to the virtuality network shown in Figure 2, there are a number of other possible illusions involving the interactions between the peripheral nervous system and the central nervous system. This is particularly true in cases where the body is not in the same contextual frame as the visual and auditory systems.

\section{Additional Considerations}

This section will highlight additional considerations, including emerging challenges, opportunities for open science, potential future directions for research, and the increasing omnipresence of virtual media.

\section{Emerging Challenges}

There are also several technical challenges when considering the future of VR as a research tool. These challenges can be summarized as a series of trends: portability, ubiquity, and usability. Whereas these issues have been partially overcome as computing power and visual display capacity have increased, they still serve as barriers depending on how VR systems might be used.

One major challenge to using VR and neuroimaging, particularly when taking advantage of naturalistic experimental designs, is to allow for the freedom of natural behavior without the movement artifact associated with many of these behaviors. Behaviors such as human mobility (e.g., walking, driving) require portable, unobtrusive interfaces that do not distract from the cognitive task being performed. There are differences between heads-up displays and monocles in terms of how information is displayed in the visual field as well as how aware people are of the hardware. This can not only degrade the ecological validity of experimental investigations, but can also interfere with the feeling of immersion that the VR stimulus is intended to provide. 


\section{Opportunities for Open Science}

The concurrence of Virtual Neuroscience and the Open Science movement provides a number of opportunities to produce better science. I will focus on two of these: open-source content and open cognitive models. One way to ensure reproducibility and stimulus effectiveness is to contribute to and explore the opensource VR community (see Additional Information section). Developing a set of best practices for content creation within and among research groups will bolster the experimental validity of the method. Another issue mentioned previously is to develop VR-specific cognitive models. Although many detailed and even executable cognitive models exist, they are often either part of proprietary software packages or published in opaque form (e.g., non-accessible journals and programming languages). One sociology of science issue involves viewing the media-specific effects of virtual experience as epiphenomena. A focus on reproducible research and formal cognitive models may serve to mitigate skepticism from the wider community of cognitive neuroscientists.

\section{Future Directions}

One trend to follow is the explosion of data at the intersection of VR systems and neuroimaging. As VR-related equipment becomes increasingly more affordable in terms of fidelity per dollar, there will be an incentive to use such systems as experimental stimuli. This will lead to an explosion in the amount and variety of data centering on the cognitive neuroscience of virtual interactions. With this increase in data generation comes a need to adopt open science practices (Gilmore et al., 2017). For example, there is an opportunity for data to be systematically shared and reanalyzed. Whereas this provides a virtuous resource that transcends the media and communication neuroscience community, these data require investments in organization and maintenance (Poldrack \& Gorgolewski, 2014). Ultimately, look for a big data ecosystem (Khan et al., 2014) to emerge and help to improve our understanding of the major scientific issues. The use of big data in VR will be unique in that VR can serve as both the stimulus that enables data collection and an environment to visualize, evaluate, and meta-analyze the archived data (Matthews, 2018).

Virtual media omnipresence. Another trend to consider is the omnipresence of media content via $V R$ and $A R$ interfaces. As virtual and augmented reality equipment become more affordable and easy to wear, they will become integrated into our daily lives and social interactions. Although we may not notice the distinction between real and virtual objects in such a world, there will be new sets of effects and behaviors nonetheless. Perhaps we will one day speak of a new field-specific term: "ubiquitous virtuality," or the neuroscience of ever-present media. 
A third trend will involve exploring longer-term and global media effects. This is particularly the case as the effects of virtuality are experienced over longer durations of time by a more diverse group of people (e.g., non-WEIRD populations; see Henrich et al., 2010). As virtual technologies diffuse across the world and into various cultural/demographic groups, new effects will emerge that interface not only with cognitive processing, but also the cultural and biological components of global human variation.

Historical serendipity. It is an opportune time to study the effects of VR on human nervous systems, as relatively affordable, easy-to-use systems are now becoming mainstream consumer goods and work tools. Using historical trends for Google Scholar search terms, Hamacher et al. (2016) have shown that the terms "virtual reality" and "mixed reality" started to grow exponentially in the early 1990s. This growth peaked around 2010 and resulted in a trough centered on the year 2014. Whereas these trends are currently on the increase, the search term "augmented reality" shows a slightly different trend: exponential increase from 2000 to 2014, with a more recent slight decline. In both cases, it is clear that communication neuroscience can take advantage of this robust growth. It is also clear that the use of VR in neuroscience is supported by a growing bibliography of prior studies. The Gartner report on technology hype cycles (Gartner Consulting, 2016) paints an even more encouraging picture: VR resides along the slope of enlightenment and AR along the trough of disillusionment. Both are expected to arrive on the plateau of productivity in roughly 5-10 years. With the introduction of easy-to-use consumer hardware and open-source software, using VR for research has never been more accessible.

\section{Additional Information}

Here are a few examples of open source resources to get you started in creating and implementing virtual stimuli, as well as taking advantage of open datasets:

Open Source Virtual Stimulus \#1: Asteroid Impact: https://github.com/richardhuskey/ asteroid impact

Open Source Virtual Stimulus \#2: Space Fortress 5: https://github.com/CogWorks/SpaceFortress

Open Source Content Generator \#1: Mozilla A-frame: https://aframe.io/ 
Open Source Content Generator \#2: Open Space 3D: http://www.openspace3d.com

Open Data Source \#1: Open Neuro: https://openneuro.org/

Open Data Source \#2: Open fMRI: https://openfmri.org/

\section{References}

Alicea, B. (2015). Animal-oriented virtual environments: Illusion, dilation, and discovery. F1000 Research, 3, 202.

Balconi, M. \& Vanutelli, M.E. (2017). Cooperation and competition with hyperscanning methods: Review and future application to emotion domain. Frontiers in Computational Neuroscience, 11, article 86.

Baños, R.M., Botella, C., Garcia-Palacios, A., Villa, H., Perpina, C., \& Alcaniz, M. (2000). Presence and reality judgment in virtual environments: A unitary construct? CyberPsychology and Behavior, 3, 327-335.

Barron, A.B., Gurney, K.N., Meah, L.F.S., Vasilaki, E., \& Marshall, J.A.R. (2015). Decisionmaking and action selection in insects: Inspiration from vertebrate-based theories. Frontiers in Behavioral Neuroscience, 9, article 216.

Bartels, A. \& Zeki, S. (2004). The chronoarchitecture of the human brain-Natural viewing conditions reveal a time-based anatomy of the brain. Neurolmage, 22, 419-433.

Beggs, J.M. (2008). The criticality hypothesis: How local cortical networks might optimize information processing. Philosophical Transactions Royal Society A, 366, 329343.

Biocca, F. \& Delaney, B. (1995). Immersive virtual reality technology. In F. Biocca \& M. Levy (Eds,), Communication in the age of virtual reality (pp. 57-124). Hillsdale, NJ: Lawrence Erlbaum Associates.

Bohil, C., Alicea, B., \& Biocca, F. (2011). Virtual reality in neuroscience research and therapy. Nature Reviews Neuroscience, 12, 752-762.

Bystrom, K.-E., Barfield, W., \& Hendrix, C. (1999). A conceptual model of the sense of presence in virtual environments. Presence, $89,241-244$. 
Chance, S. S., Gaunet, F., Beall, A. C., \& Loomis, J. M. (1998). Locomotion mode affects the updating of objects encountered during travel: The contribution of vestibular and proprioceptive inputs to path integration. Presence, 7, 168-178.

Chapman, H.L., Eramudugolla, R., Gavrilescu, M., Strudwick, M.W., Loftus, A., Cunnington, R., \& Mattingley, J.B. (2010). Neural mechanisms underlying spatial realignment during adaptation to optical wedge prisms. Neuropsychologia, 48, 25952601.

Cheetham, M. (2017). The Uncanny Valley Hypothesis and beyond. Frontiers in Psychology, 8, article 1738.

Csikszentmihalyi, M. (1990). Flow: The psychology of optimal experience. New York, NY: Harper \& Row.

Ehrsson, H.H., Holmes, N.P., \& Passingham, R.E. (2005). Touching a rubber hand: Feeling of body ownership is associated with activity in multisensory brain areas. Journal of Neuroscience, 25, 10564-10573.

Fulvio, J.M. \& Rokers, B. (2017). Use of cues in virtual reality depends on visual feedback. Scientific Reports, 7, 16009.

Gartner Consulting. (2016). Gartner hype cycle for emerging technologies. Retrieved from https://www.gartner.com/smarterwithgartner/3-trends-appear-in-the-gartnerhype-cycle-for-emerging-technologies-2016/

Geldard, F.A. (1982). Saltation in somesthesis. Psychological Bulletin, 92, 136-175.

Gerrig, R. J. (1993). Experiencing narrative worlds. New Haven, CT: Yale University Press.

Gibson, J.J. (2015). The ecological approach to visual perception. New York, NY: Psychology Press.

Gilmore, R.O., Diaz, M.T., Wyble, B.A., \& Yarkoni, T. (2017). Progress toward openness, transparency, and reproducibility in cognitive neuroscience. Annals of the New York Academy of Sciences, 1396, 5-18. 
Gonzalez-Franco, M. \& Lanier, J. (2017). Model of illusions and virtual reality. Frontiers in Psychology, 8, article 1125.

Good, J.M.M. (2007). The affordances for social psychology of the ecological approach to social knowing. Theory and Psychology, 17, 265-295.

Green, C.S. \& Bavelier, D. (2007). Action-video-game experience alters the spatial resolution of vision. Psychological Science, 18(1), 88--94.

Green, C.S. \& Bavelier, D. (2012). Learning, attentional control, \& action video games. Current Biology, 22, R197-R206.

Hamacher, A., Kim, S-J., Cho, S-T., Pardeshi, S., Lee, S-H., Eun, S-J., \& Whangbo, T-K. (2016). Application of virtual, augmented, and mixed reality to urology. International Neurourology Journal, 20, 172-181.

Hambrick, D.Z., Oswald, F.L., Altmann, E.M., Meinz, E.J., Gobet, F., \& Campitelli, G. (2014). Deliberate practice: Is that all it takes to become an expert? Intelligence, 45, 3445.

Hardiess, G., Meilinger, T., \& Mallot, H.A. (2015). Virtual reality and spatial cognition. In J. D. Wright (Ed.), International encyclopedia of social and behavioral sciences (pp. 133137). Amsterdam, The Netherlands: Elsevier.

Hasson, U., Landesman, O., Knappmeyer, B., Vallines, I., Rubin, N., \& Heeger, D.J. (2008). Neurocinematics: The neuroscience of film. Projections, 2, 1-26.

Hasson, U. \& Honey, C.J. (2012). Future trends in neuroimaging: Neural processes as expressed within real-life contexts. Neuroimage, 62, 1272-1278.

Henrich, J., Heine, S.J., \& Norenzayan, A. (2010). The weirdest people in the world? Behavioral and Brain Sciences, 33, 61-83.

Huskey, R., Craighead, B., Miller, M.B., \& Weber, R. (2018). Does intrinsic reward motivate cognitive control? A naturalistic-fMRI study based on the synchronization theory of flow. Cognitive, Affective, and Behavioral Neuroscience. Advance online publication. 
Huster, R.J., Mokom, Z.N., Enriquez-Geppert, S., \& Herrmann, C.S. (2014). Braincomputer interfaces for EEG neurofeedback: Peculiarities and solutions. International Journal of Psychophysiology, 91, 36-45.

Khan, N. et.al (2014). Big data: Survey, technologies, opportunities, and challenges. Scientific World Journal, 14, 712826.

Kim, T. \& Biocca, F. (1997). Telepresence via Television: Two Dimensions of Telepresence May Have Different Connections to Memory and Persuasion. Journal of Computer-Mediated Communication, 3, article 325.

Kozma, R. \& Freeman, W.J. (2017). Cinematic operation of the cerebral cortex interpreted via critical transitions in self-organized dynamic systems. Frontiers in Systems Neuroscience, 11, article 10.

Martens, U., Wahl, P., Hassler, U., Friese, U., \& Gruber, T. (2012). Implicit and explicit contributions to object recognition: Evidence from rapid perceptual learning. PLoS One, 7, e47009.

Matthews, D. (2018). Virtual-reality applications give science a new dimension. Nature, $557,127-128$.

McLuhan, M. (1964). Understanding media. New York, NY: McGraw-Hill.

Myers, P.L., Starr, A.W., \& Mullins, K. (2018). Flight simulator fidelity, training transfer, and the role of instructors in optimizing learning. International Journal of Aviation, Aeronautics, and Aerospace, 5, article 6.

Norman, D. (2008). Signifiers, not affordances. ACM Interactions, 15, 18-19.

Olson, B. (2017, February 3). It's not you, it's the interface. Retrieved from https://medium.com/@Ben.Olson/affordances-and-signifiers-in-mobile-interfacedesign-cf584696cda8.

Ozaki, T. (2012). Time series modeling of neuroscience data. New York, NY: CRC Press.

Poldrack, R.A. \& Gorgolewski, K.J. (2014). Making big data open: Data sharing in neuroimaging. Nature Neuroscience, 17, 1510-1517. 
Rangel, A., Camerer, C., \& Montague, P.R. (2008). A framework for studying the neurobiology of value-based decision making. Nature Reviews Neuroscience, 9, 545556.

Rauter, G., Sigrist, R., Koch, C., Crivelli, F., van Raai, M., Riener, R., \& Wolf, P. (2013). Transfer of complex skill learning from virtual to real rowing. PLoS One, 8, e82145.

Reeves, B. \& Nass, C. (1996). The media equation: How people treat computers, television, and new media like real people and places. Cambridge, England: Cambridge University Press.

Reggente, N., Essoe, J. K-Y., Aghajan, Z.M., Tavakoli, A.V., McGuire, J.F., Suthana, N.A., \& Rissman, J. (2018). Enhancing the ecological validity of $\mathrm{fMRI}$ memory research using virtual reality. Frontiers in Neuroscience, 12, article 408.

Regia-Corte, T., Marchal, M., Cirio, G., \& Lecuyer, A. (2013). Perceiving affordances in virtual reality: Influence of person and environmental properties in perception of standing on virtual grounds. Virtual Reality, 17, 17-28.

Saleh, G.M. et.al (2013). The development of a virtual reality training programme for ophthalmology: Repeatability and reproducibility. Eye, 27, 1269-1274.

Selfridge, O.G. (1959). Pandemonium: A paradigm for learning. Proceedings of the Symposium on Mechanisation of Thought Processes, 1959, 511-529.

Senna, I., Maravita, A., Bolognini, N., \& Parise, C.V. (2014). The marble-hand illusion. PLoS One, 9, e91688. Seth, A. K. (2007). The ecology of action selection: Insights from artificial life. Philosophical Transactions Royal Society B, 362, 1545-1558.

Sidarus, N., Vuorre, M., \& Haggard, P. (2017). How action selection influences the sense of agency: An ERP study. Neurolmage, 150, 1-13.

Simons, D.J., Boot, W.R., Charness, N., Gathercole, S.E., Chabris, C.F., Hambrick, D.Z., \& Stine-Morrow, E.A.L. (2016). Do "brain-training" programs work? Psychological Science in the Public Interest, 17, 103-186. 
Slater, M., \& Sanchez-Vives, M.V. (2016). Enhancing our lives with immersive virtual reality. Frontiers in Robotics and Al: Virtual Environments, 3, article 74.

Slater, M. (2009). Place illusion and plausibility can lead to realistic behaviour in immersive virtual environments. Philosophical Transactions Royal Society B, 364, 35493557.

Steuer, J. (1992). Defining virtual reality: Dimensions determining telepresence. Journal of Communication, 42, 73-93.

Tarr, B., Slater, M., \& Cohen, E. (2018). Synchrony and social connection in immersive virtual reality. Scientific Reports, 8, article 3693.

Teo, W-P. et.al (2016). Does a combination of virtual reality, neuromodulation and neuroimaging provide a comprehensive platform for neurorehabilitation? A narrative review of the literature. Frontiers in Human Neuroscience, 10, article 284.

Thompson, J.J., Blair, M.R., Chen, L., \& Henrey, A.J. (2013). Video game telemetry as a critical tool in the study of complex skill learning. PLoS One, 8, e75129.

Urgen, B.A., Kutas, M., \& Saygin, A.P. (2018). Uncanny valley as a window into predictive processing in the social brain. Neuropsychologia, 114, 181-185.

Weber, R., Alicea, B., Huskey, R., \& Mathiak, K. (2018). Network dynamics of attention during a naturalistic behavioral paradigm. Frontiers in Human Neuroscience, 12, article 182.

West, G.L. et.al (2017). Impact of video games on plasticity of the hippocampus. Molecular Psychiatry, 23, 1566-1574. 